\title{
INTERACTIVE VOICE RESPONSE (IVR) ELECTRONIC CONTROL UNIT (ECU) FOR UNMANNED GROUND VEHICLE (UGV)
}

\author{
Tubonimi Jenewari ${ }^{1}$, Biobele Alexander Wokoma ${ }^{2}$ \\ ${ }^{1}$ Lecturer, Dept. of Elect and Computer Engg, Rivers State University of Sci \& Tech, Rivers State, Nigeria \\ ${ }^{2}$ Grad assistant, Dept. of Elect and Computer Engg, Rivers State University of Sci \& Tech, Rivers State, Nigeria
}

\begin{abstract}
Remote operation has almost gained its ground, but more research in the area, is giving rise to further enhancement which is not an exception in unmanned ground vehicle (UGV). The sophistication of $U G V$ is increasing every day but the voice interaction of these UGV's is somehow less obvious. In military and disaster recovery, UGV's will be very busy in the field. It is important to speak out the status of key sensors while the remote operator is busy. It has been observed, that most UGV's go down without knowing the cause of the problem, though this cause can be known after recalling past event, but the damage will cause setback that will be difficult to recover from. Since the UGV is operated remotely, the feedbacks received will help guide the operator in maneuvering, knowing status of vehicle and other systems attached to it for proper decision making. The aim of the this work is to come up with a design of IVR-ECU, that will be able to communicate with other ECU's, to provide voice interaction for remote operators. This is important to complement existing graphic user interface (GUI), which is also helpful for those that have eye impediment. The ECU's will be multiplexed to the IVR-ECU. Each sensor will be tagged, and the signal will have corresponding voice response via its ECU commanded by the IVR-ECU. When requested the sensor status internal text format will speak out output in real-time; eg give advice, warn for any eminent inherent danger pose by the vehicle etc.
\end{abstract}

Key Words: Unmanned-ground-vehicle (UGV), interactive-voice-response (IVR), electronic-control-unit (ECU), textto-speech, speech synthesizer, CAN-BUS, IoT, M2M.

\section{INTRODUCTION}

Unmanned vehicle is the in-thing in today's military operations, hazard area monitoring, transport system etc. UGV's are not expected to have drivers onboard but rather remotely, this does not remove the fact of passengers not being in the vehicle, as in transportation system and in disaster recovery and evacuation. Since the UGV is operated remotely, the feedbacks received will help guide the operator in maneuvering, knowing the status of the vehicle and other systems attached to the vehicle for proper decision making. The electronics of UGV falls under autotronics, it has difference subsystems. In this light it is important to explore the existing technology and proffer modern technology on how voice communication and text-to-speech can enhance UGV's performance.

The instrumentation and software architecture of the vehicle platform, communication links, remote control station, and human-machine interface are essential parts in UGV's[1].A huge progress has been made in recent time in UGV because of large scale integration and robot technology.

Interactive voice response (IVR) is a technology that allows a computer to interact with humans through the use of voice and DTMF tones input via keypad [2]. In this work, the operator can select the ECU to interact with, or get feedback despite its auto response.

\section{RELATED WORKS}

In realizing this design there is need to look at some technology that is existing, which will help actualize this work.

During the past decade physical object embedded with electronics, sensors, software and connectivity have exchanged information one to another in a fashion with swift response. In internet of things (IoT), objects are allowed to be sensed and controlled remotely across exiting network infrastructure, making it more possible for better integration[3].In UGV a communication network (CAN BUS) already exist that makes it possible for all the ECU's to communicate with one another, which is more like machine to machine (M2M) communication; considered to be an integral part of IoT.

M2M has existed in different forms since the advent of computer networking automation, and existed before cellular communication. It has been utilized in space technology, manufacturing, process and oil \& gas industry in the applications such as telemetry, industrial automation, SCADA[4]. It was Theodore G. Paraskevakos that first conceptualized M2M devices that combined telephony and computing [4].

Interactive voice response (IVR) has been widely used in the telephone industry. Here the system is programmed to respond to the caller's request by responding to DTMF tones 
via key pad or voice. This is also widely used in telephone banking where customers make their request by pressing several combination of keys or make speech to get their request resolved [2].

Another key technology that will make this work possible is text-to-speech (TTS) technology. Artificial production of human voice is made possible by converting text format to speech using speech synthesizer[5]. The quality of a speech synthesizer is judged by its similarity to the human voice and by its ability to be understood clearly. Visual impaired or reading disability people use this technology to listen to written works on home computer. The text to speech system comprises of both front and back end. The front end assigns phonetic transcription to each word, and divides, marks the text into prosodic units [6].

A CAN BUS is a means of communication that is made up of four wires, two for power, positive and negative while the remaining two are for data, recessive and dominant. A recessive status $(2.5 \mathrm{~V})$ is when there is no transmission of signal of bit 1 is present while the dominant status $(3.5 \mathrm{~V})$ is the transmission of 0 bit.

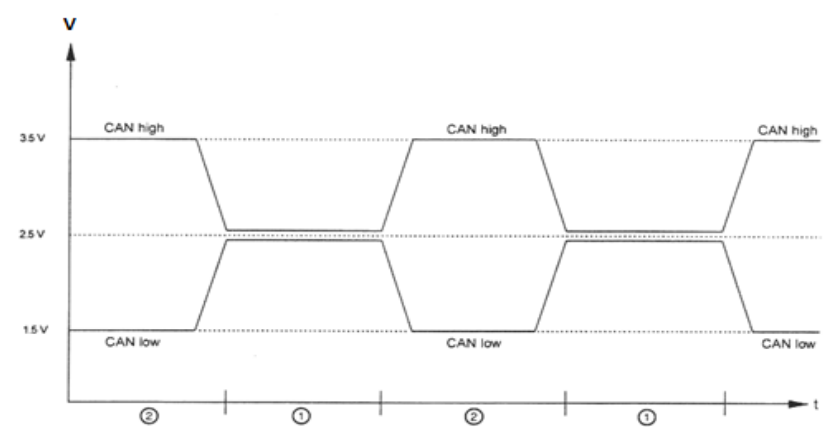

Figure 3.1: Data transfer on the CAN-Bus lines[7].

At the state of recessive status a CAN-high voltage value minus a CAN-low voltage value equals zero, which makes the control unit to automatically disconnect itself from the communication link. At the state of dominant status a CANlow voltage value equals values between $1 \mathrm{~V}$ and $5 \mathrm{~V}$ [7].

\section{METHODOLOGY}

All modern cars have electronic control units (ECU) with a combination of peripheral elements, such as sensor, transducers and actuators, which forms a subsystem responsible of managing the function and operations of vehicle system engine, light, transmission, brakes etc. the measured values provided by sensor and transducers are converted into signals that's is interpreted by microprocessor. These sensors have their signal in analog format that is converted to digital format in the ECU. The digital signal from the ECU is analyzed to generate tag/code for each sensor. The generated tag/code will be interpreted into real-time text to speech via the designed interactive voice response ECU using speech synthesizer etc.

The new CU (IVR-ECU) can easily be connected to the system via CAN BUS line.

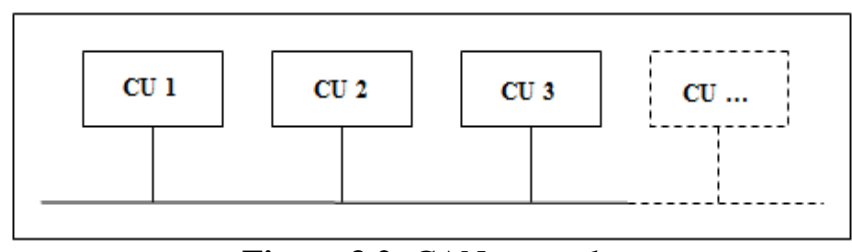

Figure 3.2: CAN network

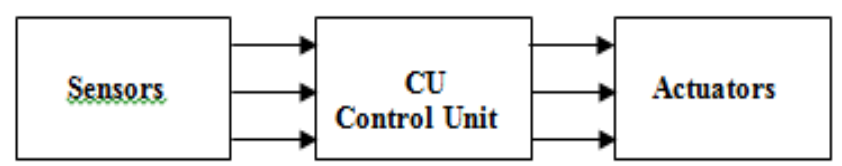

Figure 3.3: Combination of Control Unit with peripheral elements

Since there is no central control unit that decides which control unit of the network is allowed to access the Bus, the IVR-ECU will receive the transmitted bit on the BUS line. The structure of the CAN Bus protocol is shown in figure 3.4.

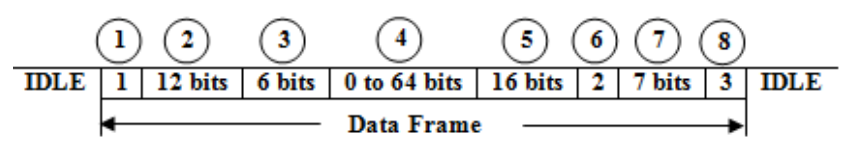

Figure 3.4: CAN-Bus protocol

1 is the start bit

2 is the arbitration field that contains the identifier

3 indicate the number of data bytes in the data field, it is known as check field.

4 is the data field

5 is the cyclic redundant check

6 is the acknowledge field

7 is the end of frame field

8 is the intermission field

Since all control units can send data at the same time, the one that has high priority in the arbitration field will be given priority, therefore the IVR-ECU will have such priority in order to access the BUS line at will.

The other part of the IVR-ECU is the conversion of information gotten from other ECU to speech using speech synthesis. Before this happens the values of each sensors is converted to digital readable text format in the IVR-ECU, this text format will then pass through text normalization, where all abbreviations are written in full form in the context of their usage in text. Once all the texts are normalized, phonetic conversion begins where all words are converted to their phonetic equivalent.

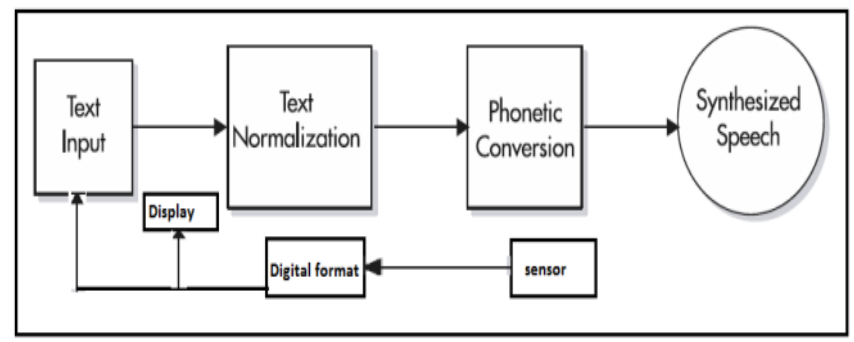

Figure 3.5: process of speech synthesis with sensors 
Request made by driver by speech to know the status of the vehicle is passed through speech recognition process. The first step in speech recognition is to capture and digitize user input. The next is to transform the digital data into sets of numbers that represent pitch combination. After this is the phonetic mapping process which renders spectral analysis information to their closest and the best possible phoneme result. Qualifier sets of phoneme strings are generated, each string has its own score that indicates its proximity to a spoken word.

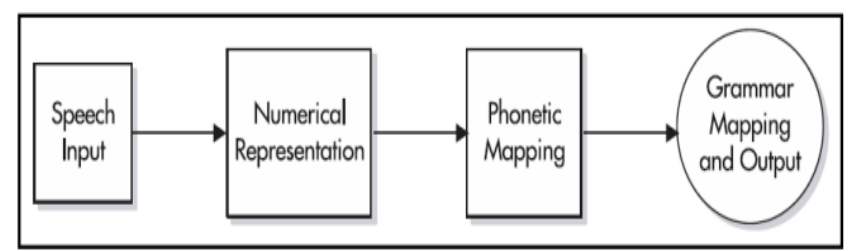

Figure 3.6: speech recognition process

\section{CONCLUSION}

This publication is based on providing means for remote operator for UGV's to have clear picture of what is happening in and around their vehicle when busy with serious field work, by designing IVR-ECU to provide text to speech real-time status report from key sensors. Request can also be made via the IVR-ECU by speech or DTMF keypad.

\section{REFERENCE}

[1]. Pekka Appelqvist, Jere Knuuttila and Juhana Ahtiainen (2010). Mechatronics Design of an Unmanned Ground Vehicle for Military Applications, Mechatronic Systems Applications, Annalisa Milella Donato Di Paola and Grazia Cicirelli (Ed.), ISBN: 978-953-307040-7, InTech, Available from: http://www.intechopen.com/books/mechatronicsystems-applications/mechatronics-design-of-anunmannedground-vehicle-for-military-applications.

[2]. Charul Shukla, Avnish Dass and Vikas Gupta. voiceXML 2.0 developers guide; Building Professional Voice-Enabled Applications with JSPTM,ASP, \& ColdFusion®Copyright (C) 2002 by The McGraw-HIll Companies, Inc

[3]. A harvard business review analytic services report internet of things: science fiction or business fact? Copyright (C) 2014 Harvard Business School Publishing.(https://hbr.org/resources/pdfs/comm/verizo n/18980_HBR_Verizon_IoT_Nov_14.pdf)

[4]. Machine to Machine (M2M) Technology in Demand Responsive Commercial Buildings; by David S. Watson, Mary Ann Piette, Osman Sezgen, and Naoya Motegi Lawrence Berkeley National Laboratory

[5]. Allen, Jonathan; Hunnicutt, M. Sharon; Klatt, Dennis (1987). From Text to Speech: The MITalk system. Cambridge University Press. ISBN 0-521-30641-8

[6]. van Santen, Jan P. H.; Sproat, Richard W.; Olive, Joseph P.; Hirschberg, Julia (1997). Progress in Speech Synthesis. Springer. ISBN 0-387-94701-9.

[7]. Arieh Nachum, Automotive CAN-Bus System TPS3531

\section{BIOGRAPHIES}

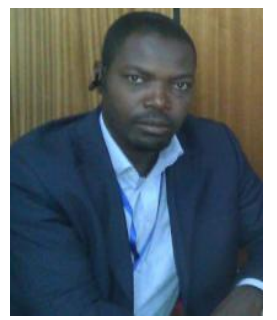

He graduated and obtained a B.Tech degree in Electronics Engineering in Rivers State University of Science \& Technology where he is working presently, after his first degree he worked for some years before proceeding for further studies.

In 2008, he obtained MSc. in mobile personal and satellite communication in the University of Westminster, UK. Within this period he also attended the Institute of Engineering and Technology and obtained a level 3 certificate in the requirement for electrical installations (BS7671 2008). $\mathrm{He}$ is enthusiastic in electronic and telecoms design and has his own small lab for PLC, embedded system, etc.

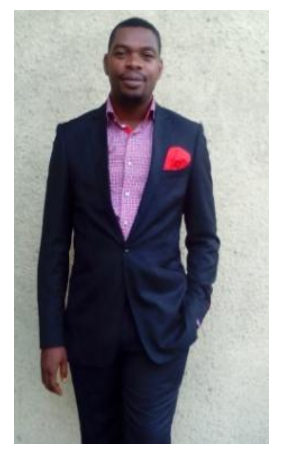

He studied and obtained a B.Tech degree in Electrical Engineering (Electronics Option) from the Rivers State University, where he works at present.

He is presently a post graduate student in the university of Port Harcourt, Rivers State, Nigeria, where he is studying Electronics \& Telecommunication Engineering (M.Eng). 